\title{
Der EuGH als Feind? Die Debatte um das soziale Europa in der europäischen Rechtsprechung
}

\author{
Franz C. Mayer*
}

Die Rechtsprechung des Europäischen Gerichtshofs (EuGH) ist immer wieder Gegenstand von Kritik gewesen. Von Anbeginn hat die durch den EuGH betriebene richterliche Rechtsfortbildung immer wieder sowohl mitgliedstaatliche Gerichte als auch die Fachöffentlichkeit herausgefordert. ${ }^{1}$ Vergleichsweise selten waren dabei allerdings Fälle, in denen eine EuGH-Entscheidung auch jenseits der Fachkreise oder gar jenseits der betroffenen Mitgliedstaaten Diskussionen ausgelöst hat. ${ }^{2}$

Mit einer Serie von Entscheidungen ab 2007 hat der EuGH nun aber das Entstehen einer bis dahin nicht gekannten breiten Front von Kritikern jenseits des europarechtlichen Fachdiskurses und jenseits der durch die Urteile unmittelbar betroffenen Mitgliedstaaten verursacht. ${ }^{3}$ Von einem „Generalangriff des EuGH auf Tarifautonomie und Arbeitnehmerrechte ${ }^{“ 4}$ ist die Rede, Grundrechte und ,,auch die Unabhängigkeit der Gewerkschaften“ seien ,,in Gefahr", 5 es drohe ,[d]er europäische Weg zur Knechtschaft“, weil ,,der Europäische Gerichts-

* Prof. Dr. jur. Franz C. Mayer, LL.M. (Yale), Lehrstuhl für Öffentliches Recht, Europarecht, Völkerrecht, Rechtsvergleichung und Rechtspolitik, Fakultät für Rechtswissenschaft, Universität Bielefeld. Aktualisierte Fassung eines Beitrages für das Expertengespräch „Der EuGH und das soziale Europa“ am 2. Februar 2009 bei der Friedrich-Ebert-Stiftung. Siehe auch Friedrich-Ebert-Stiftung (Hrsg.): Der EuGH und das soziale Europa. Für eine Aufwertung sozialer Grundrechte im EU-Rechtssystem, Internationale Politikanalyse, Berlin 2009.

1 Besonders kontrovers waren beispielsweise die Rechtsprechung zur unmittelbaren Anwendbarkeit von Richtlinien bzw. zur mitgliedstaatlichen Haftung bei Nichtumsetzung von Richtlinien oder der Ausbau der Berechtigungen aufgrund des Unionsbürgerstatus.

2 Ein jüngeres Beispiel ist hier die EuGH-Entscheidung zur Zulassung von Unionsbürgern zum Studium in Österreich, die jedenfalls in Österreich intensiv diskutiert wurde. EuGH Rs. C-147/03 (Kommission/Österreich), Slg. 2005, I-2529.

3 Zur Kritik an den Urteilen, Viking ', ,Laval', ,Rüffert" und ,Kommission/Luxemburg ' siehe insbesondere die Nachweise bei Thomas Blanke: Die Entscheidungen des EuGH in den Fällen Viking, Laval und Rüffert - Domestizierung des Streikrechts und europaweite Nivellierung der industriellen Beziehungen, Oldenburger Studien zur Europäisierung und zur transnationalen Regulierung Nr. 18/2008 u. a. auf Sophie Robin-Olivier: Liberté de l'action syndicale $v s$ liberté d'établissement, in: Revue de Droit du Travail 1/2008, S. 8-11; Christian Joerges/Florian Rödl: Das Soziale Defizit des Europäischen Integrationsprojekts, in: Kritische Justiz 2008, S. 149-165; Brian Bercusson: The decision of the European Court of Justice in Viking: Paper presented to Catelene Passchier, European Trade Union Confederation (ETUC) Confederal Secretary, 11.12.2007; Brian Bercusson: The decision of the European Court of Justice in Laval: A preliminary analysis, paper presented to the European Trade Union Institute (ETUI) Research Group on Transnational Trade Union Rights, 09.01.2008; Thomas Blanke: Thesis with regard to the decision of the ECJ in the cases Viking and Laval, paper presented to the European Trade Union Institute (ETUI) Research Group on Transnational Trade Union Rights, 16.03.2008; Wolfgang Däubler: Gestaltungsspielräume aus deutscher Sicht, abrufbar unter: http://www.bmas.de/coremedia/generator/27056/property=pdf/2008_07_16_symposium_eugh__(letzter Zugriff: 17.06.2009); Filip Dorssemont: The right to take collective action versus fundamental economic freedoms after Laval and Viking, paper presented to the European Trade Union Institute (ETUI) Research Group on Transnational Trade Union Rights, 16.03.2008; Europäischer Gewerkschaftsbund: Stellungnahme des EGB zu den Urteilen des EuGH in den Fällen Viking und Laval. Entschließung angenommen vom EGB-Exekutivausschuss auf seiner Sitzung am 4. März in Brüssel E.179, EGB/EG179/CP/SP/em, 26.02.2008, S. 3; Eva Kocher: Kollektivverhandlungen und Tarifautonomie - Welche Rolle spielt das europäische Recht?, in: Arbeit und Recht 2008, S. 13-18.

4 Janeta Mileva/Michael Popp: Generalangriff des EuGH auf Tarifautonomie und Arbeitnehmerrechte - Hintergrundpapier zu den Urteilen Laval, Viking und Rüffert, herausgegeben von DIE LINKE im Deutschen Bundestag, abrufbar unter: http://dokumente.linksfraktion.net/pdfmdb/7798171565.pdf (letzter Zugriff: 17.06.2009).

5 Europäischer Gewerkschaftsbund: Stellungnahme des EGB zu den Urteilen des EuGH in den Fällen Viking und Laval, 2008. 
hof das Streikrecht aushebelt““, ${ }^{6}$ der EuGH „,schränkte das Streikrecht ein“ und sei durch seine „reaktionären Urteile“ „Vorreiter der Missachtung politischer und sozialer Grundrechte“, 7 es wird sogar zum Rechtsbruch aufgerufen: „Der einzige Weg ist, dem EuGH nicht zu folgen“. ${ }^{8}$ Ein ,Freifahrschein für soziales Dumping“ wird ausgemacht, ${ }^{9}$ vom ,Skandalösen dieser Urteile“ geredet, ${ }^{10}$ dem EuGH unterstellt, ihm schwebe ,ein uniformes, sowjetisiertes Modell industrieller Beziehungen“" vor, ${ }^{11}$ überhaupt wird dem EuGH ganz offenbar eine Art geheimer Angriffsplan („Kampfansage“) zur Vernichtung der Gewerkschaften oder Schlimmeres unterstellt, wenn er unter „Usurpation von Zuständigkeiten“ „ein gewagtes strategisches Experiment" durchführt. ${ }^{12}$ Sogar in den Klagen der Partei DIE LINKE gegen den Vertrag von Lissabon vor dem Bundesverfassungsgericht wurden die einschlägigen Urteile - die ja das geltende Recht betreffen, nicht den Vertrag von Lissabon - thematisiert, allerdings ohne jeden Erfolg. ${ }^{13}$

Wer all dies und Ähnliches liest gruselt sich mehr und mehr und fragt sich irgendwann, ob diese schwerwiegenden Vorwürfe und dramatischen Einordnungen wirklich zutreffend sein können. ${ }^{14}$

Einen ersten Hinweis darauf, dass die Dinge etwas nüchterner betrachtet werden können und dann auch differenzierter eingeordnet werden sollten, ergibt bemerkenswerterweise der Blick in die betroffenen Staaten. Dort ist die Diskussion zum Teil sehr viel ruhiger als in Staaten, die kaum oder gar nicht von den Urteilen berührt werden. Dies belegt das Beispiel Schweden, soweit man sich dort schlicht überlegt, wie man die Vorgaben des Europarechts am besten umsetzt. ${ }^{15}$

In der Kritik stehen die EuGH-Entscheidungen in den Rechtssachen ,Viking ‘ und ,Laval ‘ aus dem Dezember $2007^{16}$ zur Vereinbarkeit von nationalem kollektiven Arbeitsrecht mit europarechtlichen Vorgaben ${ }^{17}$ sowie die Urteile ,Rüffert ${ }^{\circ 18}$ und ,Kommission gegen Lu-

6 Klaus Dräger/Janeta Mileva: Der europäische Weg zur Knechtschaft. Wie der Europäische Gerichtshof das Streikrecht aushebelt, in: Sozialismus 7-8/2008, S. 28-33.

7 DIE LINKE: Frieden, Demokratie, Solidarität - Für ein besseres Europa. Parteivorstand DIE LINKE - Entwurf für ein Europawahlprogramm, 08.10.2008.

8 „Der einzige Weg ist, dem EuGH nicht zu folgen“, Interview mit Fritz Scharpf, in: Magazin Mitbestimmung 7$8 / 2008$, S. 18-23.

9 Wie weiter mit dem sozialen Europa? Kommentare zu Fritz Scharpfs EuGH Kritik von Hans-Joachim Schabedoth, Gero Maaß, Otto Jacobi und Wolfgang Kowalsky, in: Magazin Mitbestimmung 9/2008, S. 53-57, hier S. 57.

10 Ebenda.

11 Ebenda.

12 Zitate bei Martin Höppner: Usurpation statt Delegation. Wie der EuGH die Binnenmarktintegration radikalisiert und warum er politischer Kontrolle bedarf, MPIfG Discussion Paper 08/12, S. 30.

13 BVerfG, Urteil vom 30.06.2009 - Lissabon, Absatz-Nr. 392ff. Wörtlich heißt es: „Die von den Beschwerdeführern [...] vorgetragene Behauptung, die europäische Wirtschaftspolitik sei reine Marktpolitik ohne sozialpolitische Ausrichtung und beschränke mit ihrem funktionellen Ansatz die Möglichkeiten der Gesetzgebung in den Mitgliedstaaten, eine selbstbestimmte Sozialpolitik zu betreiben, ist unzutreffend. Weder ist die Europäische Union sozialpolitisch ohne Kompetenzen, noch ist sie auf diesem Gebiet untätig. Zugleich verfügen die Mitgliedstaaten über einen ausreichenden Zuständigkeitsraum, um sozialpolitisch wesentliche Entscheidungen selbstverantwortlich zu treffen."

14 Lüder Gerken (mit Roman Herzog) äußert immerhin in der in weiten Teilen unsachlichen EuGH-kritischen Polemik „Stoppt den Europäischen Gerichtshof!“, in: Frankfurter Allgemeine Zeitung, 08.09.2008, S. 8, ausgerechnet an den hier in Rede stehenden EuGH-Urteilen keine Kritik.

15 Siehe dazu das Regierungsmemorandum Action in response to the Laval judgment, SOU [Swedish Government Official Reports] 2008:123.

16 EuGH Rs. C-438/05 (Viking), Slg. 2007, I-10779; EuGH Rs. C-341/05 (Laval), Slg. 2007, I-11767.

17 Vgl. hierzu Christian Joerges/Florian Rödl: Von der Entformalisierung europäischer Politik und dem Formalismus europäischer Rechtsprechung im Umgang mit dem „sozialen Defizit“ des Integrationsprojekts. Ein Beitrag aus Anlass der Urteile des EuGH in den Rechtssachen Viking und Laval, ZERP-Diskussionspapier 2/2008.

18 EuGH Rs. C-346/06 (Rüffert), Slg. 2008, I-1989. 
xemburg ${ }^{6}{ }^{19}$ in denen Tariftreueklauseln im Vergaberecht beziehungsweise eine nationale Umsetzungsgesetzgebung der Entsenderichtlinie für gemeinschaftsrechtswidrig erklärt wurden.

Die beanstandeten Entscheidungen lassen sich im Themenfeld ,Soziales Europa 'verorten. Ein Kernvorwurf an den EuGH ist dabei, er berücksichtige die sozialen Verpflichtungen der Europäischen Union in seinen Entscheidungen im Verhältnis zu den Wirtschaftsfreiheiten nicht angemessen und trage damit nicht zur Behebung eines ,sozialen Defizits ' des Integrationsprojekts bei. In die Kritik an dieser Rechtsprechung mischen sich Einwände gegen den Vertrag von Lissabon, dem - wie bereits dem Verfassungsvertrag - Marktradikalismus vorgeworfen wird. ${ }^{20}$ Was genau sich mit sozialem Europa verbindet, ist dabei nicht immer eindeutig bestimmt. ${ }^{21}$ In diesen Kontext lassen sich Fragen der Harmonisierung oder Koordinierung nationalen Arbeits- oder Sozialrechts ebenso bringen wie Fragen der Beschäftigungspolitik, der Daseinsvorsorge oder der nicht-ökonomischen Marktschranken.

Die Debatte um ein soziales Europa wird dabei nicht selten mit der Frage vermengt, wie der EuGH sich zur sozialen Dimension der europäischen Integration verhält. Im Folgenden geht es vorrangig um diese letztgenannte Frage.

Um hier eine bessere Vorstellung von der Grundlage zu erlangen, auf der die jüngst in die Kritik geratene Rechtsprechung des EuGH fußt, ist zunächst eine Vergewisserung über die Grundtendenz zum sozialen Europa in den vertraglichen Grundlagen der Europäischen Union sinnvoll. Der anschließende Rückblick in die frühere Rechtsprechung des EuGH ermöglicht es, die neueren Urteile, die die heftige Kritik auslösen, einordnen zu können. Vor diesem Hintergrund lassen sich dann Überlegungen dazu anstellen, welche Spielräume der EuGH im Hinblick auf das soziale Europa hat.

\section{Die rechtlichen Rahmenbedingungen: EG und EU als Wirtschafts- und Sozialunion}

Nicht leugnen lässt sich, dass die Gemeinschaft ursprünglich auf die Errichtung eines Binnenmarktes ausgerichtet war. Die Europäische Wirtschaftsgemeinschaft (EWG) war zunächst kein Projekt zur Errichtung eines einheitlichen gemeinsamen Sozialraums. Fritz W. Scharpf hat einmal im europäischen Kontext von der Entkopplung der sozialen Politik von der wirtschaftlichen Verfassung gesprochen. ${ }^{22}$ Diese Beobachtung darf indessen nicht dazu verleiten, der Europäischen Union jegliche soziale Dimension abzusprechen und eine schematische Gegenüberstellung ,Markt auf europäischer Ebene“ versus ,Soziales auf nationaler Ebene" vorzunehmen.

Die Reduktion auf eine bloße Wirtschaftsgemeinschaft, die ausschließlich am ökonomischen Eigennutz orientiert ist, war noch nie eine zutreffende Beschreibung der europäischen Integration. Die Europäische Union beziehungsweise Europäischen Gemeinschaften waren nie nur ,Markt‘. Bereits die Verständigung auf die Gründung der EWG 1957 kam letzten Endes nur zustande, weil von Anfang an mit der Vorgabe des heutigen Art. 141 des Vertra-

19 EuGH Rs. C-319/06 (Kommission/Luxemburg), Slg. 2008, I-4323.

20 Siehe dazu etwa die Klage der Fraktion DIE LINKE gegen den Vertrag von Lissabon vor dem Bundesverfassungsgericht (BVerfG). Auch in der irischen Debatte nach dem gescheiterten Referendum spielt das Thema eine Rolle, siehe etwa den Bericht eines Unterausschusses des irischen Parlamentes zur Zukunft Irlands in der Europäischen Union vom November 2008 (Houses of the Oireachtas, Sub-Committee on Ireland's Future in the European Union: Ireland's future in the European Union: Challenges, Issues and Options, November 2008).

21 Das in jüngerer Zeit zunehmende Ausmaß an Befassung mit dem Thema ,Soziales Europa' steht dabei in umgekehrt proportionalem Verhältnis zur Trennschärfe des Begriffs.

22 Fritz W. Scharpf: The European Social Model: Coping with the Challenges of Diversity, in: Journal of Common Market Studies 2002, S. 645-670. 
ges zur Gründung der Europäischen Gemeinschaft ${ }^{23}$ (gleiches Entgelt für Mann und Frau in der Arbeitswelt) ein Marktkorrektiv akzeptiert wurde. Die Aufnahme dieses Korrektivs geht übrigens auf Frankreich zurück, das sich in den Verhandlungen kurz vor dem Scheitern des gesamten Vertragswerkes gegen deutsche Bedenken wegen der mindestlohnartigen Vorgabe für die Entlohnung von Frauen als Eingriff in den Markt durchsetzte, weil es ansonsten einen Wettbewerbsnachteil wegen der in Frankreich bereits weiter als anderswo reichenden Lohngleichheit für Frauen befürchtete. ${ }^{24}$

Diese Konstellation, in der soziale Gesichtspunkte als Wettbewerbsnachteile erscheinen, ist ebenso paradigmatisch wie die seinerzeit von Frankreich in einer langen Verhandlungsnacht von Premierminister Guy Mollet gegen Bundeskanzler Konrad Adenauer und gegen deutschen Widerstand, namentlich aus dem Bundeswirtschaftsministerium Ludwig Erhards, erzwungene Lösung der auf europäischer Ebene festgeschriebenen Verpflichtung auf gleiche Standards für alle, soweit es um die Entlohnung von Männern und Frauen geht. Diese Lösung verdient auch vor dem Hintergrund aktuellerer Mindestlohndebatten Beachtung.

Neben dem Gebot des gleichen Entgelts für Männer und Frauen enthält das derzeitige Primärrecht an etlichen Stellen weitere Elemente, die sich als soziale Ausgestaltungen des Europarechts auffassen lassen. ${ }^{25}$ Diese sozialen Elemente der europäischen Integration im Primärrecht werden durch den Vertrag von Lissabon durchweg übernommen, vielfach verstärkt. Der Verfassungskonvent, der den Vorläufer des Vertrags von Lissabon, den Verfassungsvertrag, ausarbeitete, hatte mit der Arbeitsgruppe XI „Soziales Europa“ ${ }^{26}$ einen Anlauf unternommen, das Sozialthema im Primärrecht sichtbarer zu machen. Neben der Fortführung der sozialen Elemente aus dem geltenden Primärrecht bringt der Vertrag von Lissabon in der Verlängerung dieser Entwicklung eine Reihe von genuinen Neuerungen im Bereich soziales Europa. Beispielsweise erfolgt mit dem Vertrag von Lissabon in Art. 3 EUV-Liss. ${ }^{27}$ erstmals auch im Wortlaut des Primärrechts ein Bekenntnis zur sozialen Dimension der Marktwirtschaft (soziale Marktwirtschaft). Neben einer Reihe weiterer Änderungen am Primärrecht impliziert die Verbindlichkeit der Charta der Grundrechte (Art. 6 EUV-Liss.) ebenfalls eine Verbesserung in Sachen soziales Europa. Damit werden nämlich erstmals auch soziale Grundrechte ${ }^{28}$ im Primärrecht verankert, diese finden sich in der Charta im Kapitel IV „Solidarität“. In der Präambel der Charta wird der Grundsatz der Solidarität als gleichrangiger Wert neben Werten wie Menschenwürde, Freiheit, Gleichheit, Demokratie und Rechtsstaatlichkeit genannt.

Insgesamt ergibt sich, dass das geltende und das künftige Primärrecht keineswegs eine einseitige Orientierung des europäischen Integrationsprozesses am Marktprinzip erkennen lassen. Die Konzeptualisierung der sozialen Gehalte im Europarecht ist bis hin zu einer Beschreibung von Solidarität als Leitprinzip der Verträge unternommen worden, ${ }^{29}$ was in die

23 Konsolidierte Fassung des Vertrags über die Europäische Union (EUV) und des Vertrags zur Gründung der Europäischen Gemeinschaft (EGV) in der Fassung des Vertrages von Nizza, in: Amtsblatt der EU, Nr. C 321 E vom 29. Dezember 2006, S. 1-331.

24 Dass es hier letztlich um eine Mindestlohnfrage geht, führt auf die Frage, ob eine solche europaweite Festschreibung eines bestimmten Lohnstandards heute denkbar wäre.

25 Siehe im Einzelnen Franz C. Mayer: Der EuGH und das soziale Europa, in: Friedrich-Ebert-Stiftung (Hrsg.): Der EuGH und das soziale Europa. Für eine Aufwertung sozialer Grundrechte im EU-Rechtssystem, Internationale Politikanalyse, Berlin 2009, S. 7-25.

26 Europäischer Konvent: Schlussbericht der Gruppe XI „Soziales Europa“, Konventdokument CONV 516/1/03 REV 1 vom 04.02.2003.

27 Konsolidierte Fassung des Vertrags über die Europäische Union (EUV-Liss.) in der Fassung des Vertrags von Lissabon, in: Amtsblatt der EU, Nr. C 115 vom 9. Mai 2008, S. 13-45.

28 Siehe zu den Hintergründen das Braibant/Meyer-Papier, Dokument CHARTE 4401/00 vom 04.07.2000.

29 Christian Tomuschat: Solidarität in Europa, in: Francesco Capotorti (Hrsg.): Du droit international au droit de l'intégration. Liber amicorum Pierre Pescatore, Baden-Baden 1987, S. 729-757, hier S. 733. 
Nähe eines Sozialstaatsprinzips führt. Zugleich ist festzuhalten, dass soziale Elemente vorrangig unter dem Aspekt der Koordinierung und des Marktkorrektivs eine Rolle spielen, weniger unter dem Aspekt einer europaweiten Harmonisierung. Dass positive Integration nicht im Vordergrund steht, mithin das Beispiel des Art. 141 EGV eher als Ausnahme erscheint, belegt das Ausweichen europäischer Politik in die rechtlich nicht recht greifbaren weichen Instrumente informeller Koordinierung (offene Methode der Koordinierung). ${ }^{30}$

\section{Rechtsprechung - das soziale Europa und der EuGH}

Der EuGH sichert nach Art. 220 EGV die Wahrung des Rechts. Er überwacht also die Anwendung der oben angesprochenen Primärrechtsgehalte. Über die bloße Anwendung des bestehenden Rechts hinaus hat er aber in seiner bisherigen Rechtsprechung auch zur Entwicklung zusätzlicher sozialer Elemente in der Union beigetragen. ${ }^{31}$

Dies betrifft zunächst die nähere Ausgestaltung der Grundfreiheiten. Daneben lässt sich nachzeichnen, wie der EuGH mit der Weiterentwicklung der Grundfreiheiten ungeschriebene Rechtfertigungsgründe zur Einschränkung dieser Grundfreiheiten entwickelt hat, von denen sich nicht wenige dem sozialen Bereich zuordnen lassen. Ferner finden sich in der Grundrechtsrechtsprechung des EuGH ebenso soziale Gehalte wie in der allgemeinen Rechtsprechung.

\section{Soziale Zielsetzung und soziale Flankierung der Freizügigkeit}

Die Personenfreizügigkeit, die zunächst als Arbeitnehmerfreizügigkeit nach Art. 39 EGV am bedeutsamsten geworden ist, lässt sich als soziales Freiheitsrecht interpretieren. Die Verordnung 1612/68 über die Freizügigkeit der Arbeitnehmer, ${ }^{32}$ mit der die Arbeitnehmerfreizügigkeit als eine der wichtigsten Grundfreiheiten konkretisiert wird, bringt dies auf den Punkt:

„Die Freizügigkeit ist ein Grundrecht der Arbeitnehmer und ihrer Familien; die Mobilität der Arbeitskräfte innerhalb der Gemeinschaft soll für den Arbeitnehmer eines der Mittel sein, die ihm die Möglichkeit einer Verbesserung der Lebens- und Arbeitsbedingungen garantieren und damit auch seinen sozialen Aufstieg erleichtern [...].“

Entsprechend dieser Logik eines Freiheitsrechtes hat der EuGH die Grundfreiheiten nicht nur als Diskriminierungsverbote aufgefasst, die die Gleichbehandlung mit den Inländern anordnen, sondern als Beschränkungsverbote, die unabhängig von einer Diskriminierung mitgliedstaatliche Maßnahmen untersagen, die die Ausübung einer Grundfreiheit wie etwa der Arbeitnehmerfreizügigkeit behindern oder weniger attraktiv machen.

Daneben hat der EuGH aber einen weiteren Aspekt der personenbezogenen Grundfreiheiten sehr früh erkannt: Niemand wird von einem Freizügigkeitsrecht Gebrauch machen, wenn die sozialen Rahmenbedingungen nicht gesichert sind. Beispiele reichen hier von der

30 Näher dazu David M. Trubek/Louise G. Trubek: Hard and Soft Law in the Construction of Social Europe: the Role of the Open Method of Co-ordination, in: European Law Journal 2005, S. 343-364.

31 Holm A. Ewert: Der Beitrag des Gerichtshofs der europäischen Gemeinschaften zur Entwicklung eines Europäischen Sozialrechts, München 1987; Manfred Zuleeg: Der Schutz sozialer Rechte in der Rechtsordnung der Gemeinschaft, in: Europäische Grundrechte-Zeitschrift 1992, S. 329-334; EuGH Rs. 126/86 (Giménez Zaera/ Instituto Nacional de la Seguridad Social), Slg. 1987, 3697 (3716).

32 Verordnung (EWG) Nr. 1612/68 des Rates vom 15. Oktober 1968 über die Freizügigkeit der Arbeitnehmer innerhalb der Gemeinschaft, in: Amtsblatt der EG, Nr. L 257 vom 19. Oktober 1968, S. 2-12. 
Gleichstellung bei Vergünstigungen für kinderreiche Familien im Bahnverkehr bis zum gleichen Zugang zu Regelungen laut BAfö ${ }^{33}$ für die Kinder von Wanderarbeitnehmern. ${ }^{34}$

Der EuGH hat die Freizügigkeit zuletzt immer wieder von der Arbeitnehmereigenschaft abgekoppelt und ausschließlich vom Unionsbürgerstatus her argumentiert, dabei aber weiter den Zugang zu sozialen Vergünstigungen in die Berechtigung einbezogen. In der Rechtssache (Rs.) ,Grzelcyk' führte dies beispielsweise zur übergangsweisen Zahlung von Sozialhilfe an einen Studenten, gestützt auf Art. 12 EGV i. V. m. dem Unionsbürgerstatus aus Art. 18 EGV. ${ }^{35}$ Man muss nicht mehr Arbeitnehmer oder Dienstleister oder Niederlassungswilliger sein, um sich auf das Europarecht berufen zu können und beispielsweise gegen Diskriminierungen aufgrund der Staatsangehörigkeit vorzugehen. Es genügt die Unionsbürgereigenschaft. In dieser Rechtsprechung zur Unionsbürgerschaft wird das Element der Solidarität betont. ${ }^{36}$

Insgesamt erscheinen damit soziale Rechte als nachgerade notwendige Ergänzung der Grundfreiheiten $^{37}$ und der Unionsbürgerschaft, sie werden vom EuGH im Dienste dieser Vorschriften gewährt. ${ }^{38}$

Abzuschichten ist all dies vom europäischen Kollisionsrecht in Fragen der sozialen Sicherheit: Auch nach der Rechtsprechung des EuGH bleiben für die Ausgestaltung der sozialen Sicherheitssysteme die Mitgliedstaaten zuständig. ${ }^{39}$ Europäisches Sozialrecht muss also zuvörderst die Vielfalt der Sozialrechtsordnungen der Mitgliedstaaten koordinieren. ${ }^{40}$ Damit ist eine gemeinschaftsrechtliche und an den Regelungszwecken des EG-Rechts ausgerichtete Auslegung der sozialrechtlichen Systembegriffe unvermeidlich. ${ }^{41}$ Dies darf aber nicht mit einer positiven Harmonisierung verwechselt werden.

33 Bundesgesetz über individuelle Förderung der Ausbildung (Bundesausbildungsförderungsgesetz - BAföG) in der Fassung der Bekanntmachung vom 6. Juni 1983 (BGBl. I S. 645, 1680), zuletzt geändert durch Artikel 2a des Arbeitsmigrationsteuerungsgesetz vom 20. Dezember 2008 (BGB1. I S. 2846).

34 Siehe dazu EuGH Rs. 152/73 (Sotgiu), Slg. 1974, 153; EuGH Rs. 32/75, (Cristini/SNCF), Slg. 1975, 1085; EuGH Rs. 137/84 (Mutsch), Slg. 1985, 2681; EuGH Rs. C-315/94 (Vos/Bielefeld), Slg. 1996, I-1417; zum Sonderfall Berufsausbildung EuGH Rs. 293/83 (Gravier), Slg. 1985, 593; EuGH Rs. 39/86 (Lair), Slg. 1988, 3161; EuGH Rs. 235/87 (Matteucci), Slg. 1988, 5589; zu den Rechten der Kinder nach Art. 12 Verordnung Nr. 1612/68 über die Freizügigkeit der Arbeitnehmer innerhalb der Gemeinschaft siehe EuGH Rs. 9/74 (Casagrande), Slg. 1974, 773; EuGH Rs. C-308/89 (Carmina di Leo), Slg. 1990, I-4185; EuGH Rs. C-7/94 (NRW/Gaal), Slg. 1995, I-1031; EuGH verb. Rs. C-11/06 (Morgan) und C-12/06 (Bucher), Slg. 2007, I-9161. Zum Sonderfall Koordinierungsrecht bei Leistungen der sozialen Sicherheit nach der Verordnung (EWG) Nr. 1408/71 des Rates vom 14. Juni 1971 zur Anwendung der Systeme der sozialen Sicherheit auf Arbeitnehmer und deren Familien, die innerhalb der Gemeinschaft zu- und abwandern, in: Amtsblatt der EG, Nr. L 149 vom 5. Juli 1971, S. 2-50 siehe etwa EuGH Rs. 67/79 (Fellinger), Slg. 1980, 535 oder EuGH, verb. Rs. C-245/94 und C-312/94 (Hoever und Zachow), Slg. 1996, I-4895.

35 Siehe hier im Einzelnen EuGH Rs. C-85/96 (Martínez Sala), Slg. 1998, I-2691; EuGH Rs. C-274/96 (Bickel und Franz), Slg. 1998, I-7637; EuGH Rs. C-184/99 (Grzelczyk), Slg. 2001, I-6193; EuGH Rs. C-413/99 (Baumbast), Slg. 2002, I-7091; EuGH Rs. C-224/98 (D'Hoop), Slg. 2002, I-6191; EuGH Rs. C-456/02 (Trojani), Slg. 2004, I-7573; EuGH Rs. C-406/04 (De Cuyper), Slg. 2006, I-6947; EuGH Rs. C-192/05 (Tas-Hagen), Slg. 2006, I-10451; EuGH Rs. C-318/05 (Kommission/Deutschland-Auslandsschulgeld), Slg. 2007, I-6957.

36 Siehe etwa EuGH Rs. C-209/03 (Bidar), Slg. 2005, I-2119; vgl. auch EuGH Rs. C-499/06 (Nerkowska), Slg. 2008, I-3993.

37 So auch Manfred A. Dauses (Hrsg.): Die neuere Rechtsprechung des Europäischen Gerichtshofs zum Arbeitsrecht, in: Neue Zeitschrift für Arbeitsrecht Beilage 3/1986, S. 11-20, hier S. 14.

38 So zur Koordinierung der nationalen Sozialversicherungssysteme, Klaus-Dieter Borchardt: Sozialrecht, in: Manfred A. Dauses: Handbuch des EU-Wirtschaftsrechts, München 2009, D.II., Rn. 64.

39 EuGH Rs. C-157/99 (Smits), Slg. 2001, I-5473; EuGH Rs. C-70/95 (Sodemare), Slg. 1997, I-3395; EuGH Rs. 238/82 (Duphar), Slg. 1984, 523.

40 Vgl. dazu Eberhard Eichenhofer: Das zum Europäischen Sozialrecht ergangene Richterrecht, in: Zeitschrift für Arbeits- und Sozialrecht 1995, S. 6-12.

41 Vgl. EuGH Rs. 53/81 (Levin), Slg. 1982, 1035, zum Arbeitnehmerbegriff; EuGH Rs. 149/79 (Kommission/Belgien), Slg. 1982, 1845, zum Begriff des öffentlichen Dienstes; EuGH Rs. 1/72 (Frilli), Slg. 1972, 457; EuGH Rs. 139/82 (Piscitello), Slg. 1983, 1427; EuGH Rs. $249 / 83$ (Hoeckx), Slg. 1985, 973, sämtlich ergangen zum Begriff der „sozialen Sicherheit“ i. S. d. Verordnung (EWG) Nr. 1408/71 des Rates vom 14. Juni 1971 zur Anwendung der Systeme der sozialen Sicherheit auf Arbeitnehmer und deren Familien, die innerhalb der Gemeinschaft zu- und abwandern. 


\section{Soziale Aspekte als Grenzen von Grundfreiheiten und Binnenmarkt}

Wird in der soeben geschilderten Rechtsprechungslinie das Soziale als Verstärker oder gar Grundlage für die weit verstandenen Grundfreiheiten aufgefasst, so lässt sich daneben eine andere Rechtsprechungslinie nachzeichnen, in der das Soziale den Grundfreiheiten entgegengestellt wird. Der EuGH hat in gleichem Maße, wie er die Reichweite der Grundfreiheiten ausgedehnt hat, auch die Möglichkeiten ausgebaut, diesen Grundfreiheiten staatlicherseits Schranken zu setzen. Es bestehen etliche ungeschriebene, durch den EuGH richterrechtlich entwickelte Rechtfertigungsgründe zur Einschränkung von Grundfreiheiten, die sich dem sozialen Bereich zuordnen lassen. Anschauliches Beispiel ist hier die Anerkennung des Schutzes der Arbeitnehmer als zwingendes Erfordernis des Allgemeinwohls zur Rechtfertigung von Beschränkungen der Grundfreiheiten durch den EuGH. Wörtlich:42 ,[...] im Recht auf Durchführung einer kollektiven Maßnahme, die den Schutz der Arbeitnehmer des Aufnahmemitgliedstaats gegen ein etwaiges Sozialdumping zum Ziel hat, [kann] ein zwingender Grund des Allgemeininteresses im Sinne der Rechtsprechung des Gerichtshofs liegen [...], der grundsätzlich eine Beschränkung einer der vom Vertrag gewährleisteten Grundfreiheiten zu rechtfertigen vermag [...].“

Weitere Beispiele für solche zwingenden Erfordernisse des Gemeinwohls, die dem Binnenmarkt Grenzen zu setzen vermögen, betreffen das finanzielle Gleichgewicht des Systems der sozialen Sicherheit, ${ }^{43}$ die Erfordernisse des Systems der Sozialhilfe ${ }^{44}$ sowie den Schutz der Sozialordnung. ${ }^{45}$

Man sollte sich bewusst machen, was diese Möglichkeit der Rechtfertigung von Beschränkungen der Grundfreiheiten genau besehen bedeutet: Es stehen sich letztlich Individuen gegenüber, die auf der einen Seite Grundfreiheiten wie etwa die Arbeitnehmerfreizügigkeit geltend machen, auf der anderen Seite Arbeitnehmerschutz wegen Sozialdumpings fordern. Und der Gerichtshof spricht im konkreten Fall letzterem - im Grundsatz - den Vorrang zu. Damit wird das an sich im Vertrag vorgesehene Verhältnis von Grundsatz (Gewährleistung von Grundfreiheiten) und Ausnahme (ausnahmsweise Rechtfertigung von Einschränkungen dieser Grundfreiheiten) nahezu umgekehrt.

Der Binnenmarkt und seine Grundfreiheiten werden demnach weder durch die Gründungsverträge noch durch den EuGH als Markt ohne Grenzen konzipiert. Grundfreiheiten haben gerade nicht automatischen Vorrang vor anderen Belangen.

\section{Soziale Grundrechte als allgemeine Rechtsgrundsätze}

Jenseits der Grundfreiheiten bestehen durch den EuGH entwickelte soziale Elemente in den richterrechtlich entwickelten allgemeinen Rechtsgrundsätzen des Gemeinschaftsrechts, zu denen der EuGH auch die Grundrechte zählt. Diese Grundrechte bestehen schon heute,

42 EuGH Rs. C-341/05 (Laval), Slg. 2007, I-11767, Rn. 103; vgl. auch EuGH Rs. C-438/05 (Viking), Slg. 2007, I10779, Rn. 77; EuGH Rs. C-165/98 (Mazzoleni und ISA), Slg. 2001, I-2189, Rn 27; EuGH verb. Rs. C-49/98, C-50/98, C-52/98 bis C-54/98 und C-68/98 bis C-71/98 (Finalarte Sociedade de Construção Civil Lda u.a.), Slg. 2001, I-7831, Rn. 33.

43 EuGH Rs. C-385/99 (Müller-Fauré und van Riet), Slg. 2003, I-4509, Rn. 73; EuGH Rs. C-157/99 (Smits und Peerbooms), Slg. 2001, I-5473, Rn. 72; EuGH Rs. C-158/96 (Kohll), Slg. 1998, I-1931, Rn. 41; EuGH Rs. C120/95 (Decker), Slg. 1998, I-1831.

44 EuGH Rs. C-70/95 (Sodemare), Slg. 1997, I-3395, Rn. 32.

45 EuGH Rs. C-275/92 (Schindler), Slg. 1994, I-1039, Rn. 58; EuGH Rs. C-67/98 (Zenatti), Slg. 1999, I-7289, Rn. 31. 
unabhängig von der Charta der Grundrechte der Europäischen Union, ${ }^{46}$ sie werden auch bei Verbindlichkeit der Charta nach Art. 6 Abs. 3 EUV-Liss. neben der Charta fortbestehen.

Schon seit den 70er-Jahren entnimmt der EuGH den Erwägungsgründen der Präambeln des EGV und heute auch des EUV, dass die Gemeinschaft nicht auf eine Wirtschaftsunion beschränkt ist, sondern auch auf eine Verbesserung der sozialen Lage abzielt. ${ }^{47}$ Für Art. 141 EGV hat dies dazu geführt, dass der Gerichtshof aus dieser Bestimmung auch Rechte für Privatpersonen ableitet, obwohl sich der Artikel seinem Wortlaut nach nur an die Mitgliedstaaten wendet. ${ }^{48}$ Dem Urteil ,Defrenne III' lässt sich entnehmen, dass dem Recht des Art. 141 EGV gar die Qualität eines Grundrechts zukommt. ${ }^{49}$

Ein jüngeres Beispiel für die Vergrundrechtlichung sozialer Gehalte ist das vom EuGH als Grundrecht konzipierte Verbot einer Diskriminierung aus Altersgründen in der Rs. ,Mangold', in der es um vereinfachte Kündigung älterer Arbeitnehmer im Kontext der Hartz-IV-Gesetze ging. ${ }^{50}$ Gerade diese Entscheidung ist übrigens heftig kritisiert worden. ${ }^{51}$ Dass der EuGH im Sozialbereich eine Gratwanderung leisten muss, ist in der anschließenden Rechtsprechung deutlich geworden, in der der Gerichtshof dem Gebot der Zurückhaltung der Gemeinschaft im Sozialbereich gegenüber den Mitgliedstaaten dadurch Rechnung trägt, dass er in der Rs. ,Bartsch“ die Reichweite der ,Mangold'-Rechtsprechung ausdrücklich klarstellt, indem er sie auf Fälle mit gemeinschaftsrechtlichem Bezug beschränkt. ${ }^{52}$

\section{Sonstige Beispiele für Sozialorientierung der Rechtsprechung}

In etlichen weiteren Zusammenhängen lässt die Rechtsprechung des EuGH eine Orientierung an sozialen Leitgedanken erkennen.

$\mathrm{Zu}$ den bekannteren Beispielen zählt in diesem Kontext die ,Francovich'-Rechtsprechung. Hier verhalf der EuGH europarechtlich vorgesehenen sozialen Vergünstigungen zur Wirksamkeit, indem er einem Arbeitnehmer bei Zahlungsunfähigkeit des Arbeitgebers einen Schadensersatzanspruch gegen den Staat zusprach, wenn dieser entgegen seiner Pflicht aus der Richtlinie über den Schutz der Arbeitnehmer bei Zahlungsunfähigkeit des Arbeitgebers, ${ }^{53}$ durch Nichtschaffung entsprechender Einrichtungen das Entstehen eines Rechts des Arbeitnehmers vereitelt hat. ${ }^{54}$

Ein anderes, weniger bekanntes Beispiel ist das EuGH-Urteil zur Betriebsübergangsrichtlinie. ${ }^{55}$ Die Richtlinie soll die Arbeitnehmer vor den Nachteilen schützen, die sich für sie aus

46 Charta der Grundrechte der Europäischen Union, in: Amtsblatt der EU, Nr. C 303 vom 14. Dezember 2007, S. 1-16.

47 EuGH Rs. 43/75 (Defrenne/Sabena), Slg. 1976, 455 (473) (Defrenne II).

48 Ebenda, 472-476.

49 EuGH Rs. 149/77 (Defrenne/Sabena), Slg. 1978, 1365 (1379) (Defrenne III).

50 EuGH Rs. C-144/04 (Mangold), Slg. 2005, I-9981, Rn. 75.

51 Siehe etwa Gerken (mit Herzog): Stoppt den Europäischen Gerichtshof!, 2008. Sehr deutlich zu Gerken und Herzog Matthias Ruffert: „Das sind verantwortungslose Brandstifter.“, Zitat in: Joachim Jahn: Karlsruhe prüft die „Hydra von Brüssel“, in: Frankfurter Allgemeine Zeitung, 29.06.2009, S. 11.

52 „Das Gemeinschaftsrecht enthält kein Verbot der Diskriminierung aus Gründen des Alters, dessen Schutz die Gerichte der Mitgliedstaaten zu gewährleisten haben, wenn die möglicherweise diskriminierende Behandlung keinen gemeinschaftsrechtlichen Bezug aufweist.“ EuGH Rs. C-427/06 (Bartsch), Urteil vom 23.09.2008, Rn. 25.

53 Richtlinie 2008/94/EG des Europäischen Parlaments und des Rates vom 22. Oktober 2008 über den Schutz der Arbeitnehmer bei Zahlungsunfähigkeit des Arbeitgebers (kodifizierte Fassung), in: Amtsblatt der EU, Nr. L 283 vom 28. Oktober 2008, S. 36-42.

54 EuGH verb. Rs. C-6/90 und C-9/90 (Francovich und Bonifaci), Slg. 1991, I-5357.

55 Richtlinie 77/187/EWG des Rates vom 14. Februar 1977 zur Angleichung der Rechtsvorschriften der Mitgliedstaaten über die Wahrung von Ansprüchen der Arbeitnehmer beim Übergang von Unternehmen, Betrieben oder Betriebsteilen, in: Amtsblatt der EG, Nr. L 61 vom 5. März 1977, S. 26-28. 
den Änderungen in den Unternehmensstrukturen ergeben können, etwa beim Übergang von Unternehmen, Betrieben oder Betriebsteilen auf einen anderen Inhaber durch vertragliche Übertragung oder durch Verschmelzung. Hier hatte der EuGH nach mehreren Vorabentscheidungsverfahren dänischer und niederländischer Gerichte zu entscheiden, ob die Richtlinie auch für die Unternehmensveräußerung im Konkursfall heranzuziehen war. In seinem Leiturteil ,Abels ${ }^{56}$ verneinte der EuGH dies unter der Erwägung, dass keine abschließende Klarheit über die Auswirkungen auf den Arbeitsmarkt bestehe, sodass sich für den Fall der Bejahung der Anwendbarkeit die ernsthafte Gefahr einer im Widerspruch zu den sozialen Zielen des Vertrags stehenden allgemeinen Verschlechterung der Lebens- und Arbeitsbedingungen der Arbeitskräfte nicht ausschließen lasse.

Ein letztes Beispiel, das auch die Grenzen der Macht des EuGH gegenüber den Mitgliedstaaten aufzeigt, ist die ,Barber'-Entscheidung, ${ }^{57}$ die die Mitgliedstaaten sogar dazu veranlasste, die Rechtsprechung des EuGH anhand des sogenannten ,Barber'-Protokolls ${ }^{58}$ zu korrigieren. ${ }^{59}$ Hier ging es um die zeitliche Beschränkung der Rückwirkung eines EuGHUrteils.

\section{Zwischenbefund}

Die Rechtsprechung des EuGH weist kein eindeutiges Ungleichgewicht im Sinne einer Ausblendung oder Reduktion der im Primärrecht vorgegebenen sozialen Elemente aus, im Gegenteil. Dass der EuGH sich in der längerfristigen Betrachtung wohl doch auf einem Mittelweg befindet, wird an der zu unterschiedlichen Zeiten von unterschiedlichen Seiten geäuBerten Kritik erkennbar. Zum einen erhielt der EuGH Beifall, ${ }^{60}$ als er urteilte, dass es nicht gegen Gemeinschaftsrecht verstoße, wenn den Reedern gestattet wird, Seeleute zu schlechteren Vergütungs- und Sozialschutzbedingungen einzustellen, als sie für nach deutschem Recht angeheuerte Seeleute gelten ${ }^{61}$ Heftig kritisiert wurde dann aber, ${ }^{62}$ dass der EuGH in der Rs. ,Paletta' die Bindung an ausländische Arbeitsunfähigkeitsbescheinigungen erklärte, ${ }^{63}$ sich für die Vergütung teilzeitbeschäftigter Betriebsratsmitglieder aussprach ${ }^{64}$ und die unterschiedliche Bezahlung von Männern und Frauen bei gleichwertiger Tätigkeit aufgrund unterschiedlicher Tarifverhandlungen kippte. ${ }^{65}$

\section{Die jüngere Rechtsprechung: ,Viking', ,Laval', ,Rüffert', ,Kommission gegen Luxemburg6}

Die vielfältig kritisierten Urteile, Viking', ,Laval‘, ,Rüffert‘ und ,Kommission gegen Luxemburg' aus den Jahren 2007 und 2008 können die Grundeinschätzung der Rechtsprechung des EuGH als in der Gesamtbetrachtung ausgewogen nicht entkräften.

56 EuGH Rs. 135/83 (Abels), Slg. 1985, 469.

57 EuGH Rs. 262/88 (Barber), Slg. 1990, I-1889.

58 Protokoll (Nr. 17) zu Art. 141 des Vertrags zur Gründung der Europäischen Gemeinschaft (1992), in: Amtsblatt der EU, Nr. C 321 E vom 29. Dezember 2006, S. 255.

59 Dazu Jörg Ukrow: Richterliche Rechtsfortbildung durch den EuGH, Baden-Baden 1995, S. 184-185.

60 Kommentar, in: Informationsdienst Europäisches Arbeits- und Sozialrecht (EuroAS) 1/1993, S. 6-7; Wolfgang Däubler: Die EG auf dem Weg zur Sozialunion? Eine Zwischenbilanz, in: Europäisches Wirtschafts- und Steuerrecht 1993, S. 272-279, hier S. 274.

61 EuGH verb. Rs. C-72/91 und C-73/91 (Sloman Neptun), Slg. 1993, I-887.

62 Bernd Schiefer: Auswirkungen der Rechtsprechung des Europäischen Gerichtshofs auf das nationale Arbeitsrecht, in: Der Betrieb 1993, S. 38-42.

63 EuGH Rs. C-45/90, (Paletta), Slg. 1992, I-3423.

64 EuGH Rs. C-360/90, (Bötel), Slg. 1992, I-3589.

65 EuGH Rs. C-127/92 (Enderby), Slg. 1993, I-5535. 


\section{,Viking ‘}

Der Rs. ,Viking '66 lag eine Vorlage aus Großbritannien zugrunde. Das finnische Fährunternehmen „Viking Line“ hatte zur Kostenersparnis das Umflaggen eines Fährschiffes von Finnland nach Estland angekündigt. Für die Beschäftigten hätte dies ungünstigere estnische Tarifverträge zur Folge gehabt. Die internationale Förderation der Gewerkschaften für Transportarbeiter („Internationale Transportarbeiter-Föderation“ - ITF) - übrigens durch die estnische Gewerkschaft unterstützt - wies als Dachverband ihre Mitgliedsgewerkschaften an, Verhandlungen mit „Viking“ zu unterlassen. Die finnische Gewerkschaft für Seeleute („Finnish Seamen“s Union“ - FSU) drohte Arbeitskampfmaßnahmen an. „Viking“ erhob in der Folge Klage in London gegen die ITF und die FSU. Die erste Instanz gab dem Antrag von „Viking“ statt und befand, die gewerkschaftlichen Arbeitskampfmaßnahmen seien Verstöße gegen die Grundfreiheiten des EGV. Die Rechtsmittelinstanz (Court of Appeal) rief den EuGH im Wege des Vorlageverfahrens an. Der Generalanwalt war der Meinung, dass Gewerkschaften Arbeitskampfmaßnahmen ergreifen dürfen, um eine Standortverlagerung zu verhindern. Der EuGH sah in den angedrohten Arbeitskampfmaßnahmen tatbestandlich eine Beschränkung der Niederlassungsfreiheit nach Art. 43 EGV. Die letztendliche Entscheidung darüber, ob die Maßnahmen der ITF und der FSU allerdings europarechtlich gerechtfertigt sind, weil sie wirklich dem Schutz der Arbeitnehmer galten und auch tatsächlich Arbeitsplätze bedroht waren, überließ der EuGH indessen dem vorlegenden Gericht. Der Streit wurde dann allerdings vor einer Entscheidung des britischen Gerichts außergerichtlich beigelegt. ${ }^{67}$

\section{,Laval`}

In der eine Woche später ergangenen EuGH-Entscheidung in der Rs. ,Laval‘68 überließ der EuGH die Frage einer Rechtfertigung nicht mehr dem vorlegenden Gericht, allerdings erkannte er zugleich das Streikrecht erstmals als europäisches Grundrecht an. Hier hatte das lettische Unternehmen „Laval“ Arbeitnehmer aus Lettland zur Durchführung von Bauarbeiten nach Schweden entsandt. Diese Konstellation fällt unter die Arbeitnehmer-Entsenderichtlinie $^{69}$ (Richtlinie 96/71). Danach können die Beschäftigungsbedingungen, die den entsandten Arbeitnehmern im Aufnahmemitgliedstaat garantiert werden, durch Rechts- oder Verwaltungsvorschriften und/oder - in der Baubranche - durch für allgemein verbindlich erklärte Tarifverträge oder Schiedssprüche festgelegt werden. Das schwedische Arbeitnehmer-Entsendegesetz präzisierte mit Ausnahme der Mindestlohnsätze die Arbeits- und Beschäftigungsbedingungen für die in der Richtlinie 96/71 aufgeführten Sachgebiete. Das Gesetz enthielt aber keine Bestimmungen über das Entgelt, dessen Festlegung in Schweden traditionell den Sozialpartnern im Wege von Kollektivverhandlungen überlassen bleibt. Damit war keine staatliche Mindestlohnregelung getroffen. Das schwedische Recht gestattet den Gewerkschaften, unter bestimmten Voraussetzungen Arbeitskampfmaßnahmen zu ergreifen, um einen Arbeitgeber zur Aufnahme von Lohnverhandlungen und zum Beitritt zu einem Tarifvertrag zu zwingen.

66 EuGH Rs. C-438/05 (Viking), Slg. 2007, I-10779.

67 Pressemitteilung der ITF vom 03.03.2008.

68 EuGH Rs. C-341/05 (Laval), Slg. 2007, I-11767.

69 Richtlinie 96/71/EG des Europäischen Parlaments und des Rates vom 16. Dezember 1996 über die Entsendung von Arbeitnehmern im Rahmen der Erbringung von Dienstleistungen, in: Amtsblatt der EU, Nr. L 18 vom 21. Januar 1997, S. 1-6. 
„Laval“" verhandelte zunächst mit der schwedischen Bauarbeitergewerkschaft über den Beitritt zum schwedischen Bautarifvertrag, schloss dann aber einen Tarifvertrag mit der lettischen Bauarbeitergewerkschaft ab, der auch die Mehrheit der Bauarbeiter bei „Laval“ angehörten. Daraufhin ergriffen schwedische Gewerkschaften Arbeitskampfmaßnahmen unter anderem in Form von Baustellenblockaden. Nach schwedischem Recht dürfen die Gewerkschaften nämlich Arbeitskampfmaßnahmen gegen einen ausländischen (nicht aber gegen einen inländischen) Arbeitgeber vornehmen, um diesen zum Beitritt zu einem Tarifvertrag zu zwingen, auch wenn der Arbeitgeber (wie „Laval“) bereits an einen anderen Tarifvertrag gebunden ist. Diese Konstellation findet sich so nicht ohne Weiteres in anderen Mitgliedstaaten. „Laval“ klagte darauf vor einem schwedischen Arbeitsgericht. Dieses Gericht legte dem EuGH die Frage vor, ob die Dienstleistungsfreiheit durch Arbeitskampfmaßnahmen dieser Art verletzt werde.

Der EuGH nahm eine Beschränkung des freien Dienstleistungsverkehrs an, die sich allerdings rechtfertigen lässt, wenn mit ihr ein berechtigtes und mit dem Vertrag zu vereinbarendes Ziel verfolgt wird und wenn sie durch zwingende Gründe des Allgemeininteresses gerechtfertigt ist.

Der EuGH wies ausdrücklich darauf hin, dass im Recht auf Durchführung einer Arbeitskampfmaßnahme, die den Schutz der Arbeitnehmer des Aufnahmemitgliedstaats gegen ein etwaiges Sozialdumping zum Ziel hat, ein solcher zwingender Grund des Allgemeininteresses (Arbeitnehmerschutz) liegen kann. In der Rs. ,Laval` hielt er jedoch auch fest, dass die Richtlinie 96/71 es dem Aufnahmemitgliedstaat nicht erlaubt, die Erbringung einer Dienstleistung in seinem Hoheitsgebiet davon abhängig zu machen, dass Arbeits- und Beschäftigungsbedingungen eingehalten werden, die über die zwingenden Bestimmungen über ein Mindestmaß an Schutz hinausgehen.

Zwar können die Mitgliedstaaten die Beachtung von Mindestlohnvorschriften mit geeigneten Mitteln durchsetzen. Entsprechende kollektive Maßnahmen (Arbeitskampfmaßnahmen) können jedoch nicht mit dem (grundsätzlich legitimen) Ziel des Arbeitnehmerschutzes gerechtfertigt werden, wenn es im aufnehmenden Staat gar keine Vorschriften zum Mindestlohn gibt. Die Aushandlung von Löhnen durch die Sozialpartner in Schweden ist eben gerade keine staatliche Mindestlohnregelung.

Der EuGH hielt ferner fest, dass die schwedische Regelung, nach der entsendende Unternehmen durch Arbeitskampfmaßnahmen zum Abschluss von Tarifverträgen gebracht werden dürfen, obwohl sie bereits in ihrem Herkunftsmitgliedstaat tarifvertraglich gebunden sind, eine Diskriminierung darstellt, da die Regelung den Gewerkschaften zugunsten schwedischer Unternehmen eine Friedenspflicht vorschreibt, und zwar sowohl wenn die schwedischen Arbeitgeber keinen Tarifvertrag abgeschlossen haben als auch wenn sie an einen anderen Tarifvertrag gebunden sind. Solche diskriminierenden Vorschriften können nur aus Gründen der öffentlichen Ordnung, Sicherheit oder Gesundheit gerechtfertigt sein. Dies war hier nicht in Sicht.

\section{,Rüffert`}

Das Urteil in der Rs. ,Dirk Rüffert/Land Niedersachsen“ erging wenige Monate später im April 2008. ${ }^{70}$ Hier ging es wieder vor allem um die Entsenderichtlinie. Der EuGH stellte fest, dass es mit der Arbeitnehmer-Entsenderichtline unvereinbar sein kann, die Vergabe eines öffentlichen Auftrags von der Verpflichtung abhängig zu machen, das am Ausführungsort tarifvertraglich vorgesehene Entgelt zu zahlen, wenn nicht von der Möglichkeit Ge-

70 EuGH Rs. C-346/06 (Rüffert), Slg. 2008, I-1989. 
brauch gemacht wurde, einen allgemeinen Mindestlohn festzulegen. Das Niedersächsische Landesvergabegesetz sieht unter anderem vor, dass Aufträge für Bauleistungen nur an solche Unternehmen vergeben werden dürfen, die sich schriftlich verpflichten, ihren Arbeitnehmern mindestens das tarifvertraglich vorgesehene Entgelt zu zahlen, diese Verpflichtung erstreckt sich auch auf Subunternehmer. Beim Bau einer Justizvollzugsanstalt beschäftigte der deutsche Bauunternehmer ein polnisches Subunternehmen, das weniger als den tarifvertraglich vorgesehenen Lohn bezahlte und es kam zu einem Rechtsstreit. Das deutsche Gericht legte dem EuGH die Frage vor, ob der freie Dienstleistungsverkehr einer gesetzlichen Regelung entgegenstehe, nach der der Zuschlag für einen öffentlichen Bauauftrag nur dann erteilt wird, wenn der Bauunternehmer sich verpflichtet, seinen Arbeitnehmern mindestens den (örtlich) vorgesehenen Tariflohn zu zahlen.

Nach der Entsenderichtlinie können die Mitgliedstaaten bei einer staatenübergreifenden Erbringung von Dienstleistungen den in anderen Mitgliedstaaten ansässigen Unternehmen unter bestimmten Voraussetzungen Mindestlohnsätze vorschreiben. Diese Voraussetzungen waren in Niedersachsen aber nicht erfüllt: Die Besonderheit der Ausgangskonstellation bestand nämlich darin, dass Niedersachsen verlangte, einen Lohnsatz aus einem nicht für allgemein verbindlich erklärten Tarifvertrag einzuhalten, obgleich die Möglichkeit der Allgemeinverbindlicherklärung in Deutschland besteht; zudem betraf die Bindung nur den öffentlichen Sektor. Damit war mit Blick auf die Dienstleistungsfreiheit eine Rechtfertigung aus Gründen des Arbeitnehmerschutzes nicht mehr gegeben; es war nämlich nicht nachweisbar, dass ein im Bausektor tätiger Arbeitnehmer nur bei seiner Beschäftigung im Rahmen eines öffentlichen Auftrags für Bauleistungen und nicht auch bei seiner Tätigkeit im Rahmen eines privaten Auftrags des Schutzes bedarf, der sich aus Mindestlohnregeln ergibt. Auch den Rechtfertigungsgrund der finanziellen Stabilität der sozialen Sicherungssysteme erkannte der EuGH zwar an, sah aber aus den übersandten Akten eine Bedrohung dieser Systeme nicht als dargetan. Er verneinte daher die Geeignetheit der Tariftreueklausel sowohl für den Schutz der Arbeitnehmer als auch für die Tarifordnung selbst deshalb, weil durch sie nur die auf Baustellen für öffentliche Stellen beschäftigten Arbeitnehmer begünstigt werden. Damit fordert der EuGH, wie beispielsweise auch schon in den Entscheidungen ,Cassis de Dijon “71 und zum Reinheitsgebot für Bier, lediglich ,gesetzgeberische Konsequenz" ${ }^{72}$

\section{,Kommission gegen Luxemburg “}

Das Urteil in der Rs. ,Kommission gegen Luxemburg ' (Entsendegesetz) ${ }^{73}$ erging Mitte 2008 in einem Vertragsverletzungsverfahren, in dem die Europäische Kommission die Umsetzung der Entsenderichtlinie in Luxemburg beanstandete. Insbesondere wurde die Geltung des luxemburgischen Arbeitsrechts für entsandte Arbeitnehmer gerügt, soweit dies auch den sozialen Mindestlohn und die automatische Anpassung jeglicher Entlohnung - also nicht nur der Mindestlöhne - an die Entwicklung der Lebenshaltungskosten umfasste. Wiederum betonte der EuGH, dass die Regelungsbestände, deren Einhaltung entsendenden Arbeitgebern durch das Empfängerland auferlegt werden dürften, durch zwingende Gründe des Allgemeininteresses zu rechtfertigen ${ }^{74}$ und in Art. 3 Abs. 1 der Entsenderichtlinie nahezu (Art. 3 Abs. 10) abschließend aufgezählt seien. ${ }^{75}$ Neben einigen anderen Regelungen verwarf der

71 EuGH Rs. 120/78 (Rewe/Bundesmonopolverwaltung für Branntwein), Slg. 1979, 649.

72 Siehe Ulrich Becker: Anmerkung zur Entscheidung Rüffert, in: Juristenzeitung 2008, S. 891-893, hier S. 893.

73 EuGH Rs. C-319/06 (Kommission/Luxemburg), Slg. 2008, I-4323.

74 Ebenda, Rn. 43.

75 Ebenda, Rn. 26, 31. 
EuGH damit die Erstreckung indexierter Löhne auf entsandte Arbeitnehmer (über den Mindestlohn hinaus) wegen Unvereinbarkeit mit der europäischen Dienstleistungsfreiheit - insbesondere weil Luxemburg in seiner Prozessstrategie versäumt hatte, dem EuGH Nachweise für zwingende Gründe des Allgemeininteresses zu liefern und mit der Berufung auf den Schutz der öffentlichen Ordnung (Art. 3 Abs. 10 der Richtlinie) - es gehe um die Wahrung des sozialen Friedens in Luxemburg - nicht durchdrang. Wörtlich heißt es: ${ }^{76}$

„Insoweit ist darauf hinzuweisen, dass der Gemeinschaftsgesetzgeber mit Art. 3 Abs. 1 Unterabs. 1 Buchst. c der Richtlinie 96/71 die Möglichkeit der Mitgliedstaaten, in die Löhne einzugreifen, auf die Mindestlohnsätze begrenzen wollte. Folglich gehört die Bestimmung des Gesetzes vom 20. Dezember 2002 betreffend die automatische Anpassung anderer Löhne als der Mindestlöhne an die Entwicklung der Lebenshaltungskosten nicht zu den Aspekten im Sinne des Art. 3 Abs. 1 Unterabs. 1 der Richtlinie 96/71.“

\section{Analyse der Urteile}

Drei Ausgangsüberlegungen sind einer Analyse der Urteile voranzustellen.

Erstens: In Anbetracht der geringen Zahl an Urteilen ist es schon deswegen verfrüht, zu der hier interessierenden Frage von einer gefestigten Rechtsprechung des EuGH auszugehen. Man wird dem EuGH noch mehr Zeit und Fallmaterial geben müssen.

Zweitens: Kennzeichnend ist fast durchgehend die Frontstellung zwischen altem Mitgliedstaat (Finnland, Schweden, Deutschland/Niedersachsen) und neuem Mitgliedstaat (Estland, Lettland, Polen). Es geht also in Wirklichkeit in den Fällen weniger um einen marktradikalen EuGH als vielmehr um ökonomische Interessengegensätze zwischen Staaten mit unterschiedlichem Lohnniveau. ${ }^{77}$ Dies lässt sich auf Gegensätze zwischen den betroffenen Individuen zurückführen, beispielsweise in der Rs. ,Rüffert ‘ beim Bau der Justizvollzugsanstalt zwischen den deutschen Bauarbeitern und den durch Subunternehmer eingesetzten polnischen Arbeitern: Es werden hier weniger die entsandten Arbeitnehmer als eher die heimischen Unternehmer vor unliebsamer Konkurrenz geschützt. ${ }^{78}$ Der Gegensatz wird besonders deutlich, wenn man sich bewusst macht, dass die polnischen Arbeiter aller Wahrscheinlichkeit nach in Niedersachsen niemals eingesetzt würden, wenn sie genauso teuer wären wie die deutschen Arbeiter. Es geht also weniger um einen ,Rechtsamok' laufenden, marktradikalen EuGH, als vielmehr um ökonomische Verteilungskämpfe, in denen die einen ein Interesse haben, ihren kompetitiven Vorteil im Lohnkostenniveau auszuspielen, die anderen genau diesen Vorteil als Existenzbedrohung wahrnehmen müssen. Die Konstellation ist aus vergangenen Erweiterungsrunden bekannt, der EuGH hat sich hier tendenziell auch in der Vergangenheit auf die Seite der schwächeren Arbeitnehmer aus den neueren Mitgliedstaaten gestellt und protektionistischen Strategien eine Absage erteilt. ${ }^{79}$

Drittens: Argumentativer Ausgangspunkt ist die aus Grundfreiheiten, namentlich der Arbeitnehmerfreizügigkeit oder der Dienstleistungsfreiheit gewährte Berechtigung - ein Individualrecht. Dieses Individualrecht wird im Dienste der Bekämpfung von Protektionismus oder noch grundsätzlicher gegen nationale Egoismen eingesetzt. Die diesem Individualrecht gegenübergestellten Gesichtspunkte wie etwa das Streikrecht oder die Regelungen der Entsenderichtlinie sind demgegenüber a priori Durchbrechungen der Grundfreiheiten und als

76 Ebenda, Rn. 47.

77 Vgl. Norbert Reich: Free Movement v. Social Rights in an Enlarged Union - the Laval and Viking Cases before the ECJ, in: German Law Journal 2008, S. 125-161, hier S. 156, 160.

78 Steffen Klumpp: Dienstleistungsfreiheit vs. Tariftreue, in: Neue Juristische Wochenschrift 2008, S. 34733477, hier S. 3475.

79 Siehe etwa EuGH Rs. C-113/89 (Rush Portuguesa Lda/Office National d'Imigration), Slg. 1990, I-1417. 
solche eng und einzelfallbezogen auszulegen. Festzuhalten ist dabei aber auch, dass der EuGH solche Durchbrechungen überhaupt zulässt. Hier ist auch auf den Unterschied zur Rechtsprechung des BVerfG aufmerksam zu machen, die beim ,Rüffert'-Fall aufzutreten scheint: Das BVerfG hatte die Verfassungswidrigkeit von Tariftreueklauseln mit den Argumenten Bekämpfung der Arbeitslosigkeit und Erhalt der finanziellen Stabilität des Systems der sozialen Sicherung sowie der Ordnungsfunktion der Tarifverträge abgelehnt. ${ }^{80}$ Das BVerfG hat aber auch nicht die Aufgabe, Protektionismus und nationale Egoismen zu bekämpfen.

Auf die an den Entscheidungen geäußerte heftige Kritik ${ }^{81}$ gibt es zunächst einmal sehr einfache Antworten: Die Festsetzung von Mindestlöhnen in den betroffenen Mitgliedstaaten, die Berücksichtigung von in den anderen Staaten getroffenen Tarifvereinbarungen (, Viking', Laval') sowie die Allgemeinverbindlicherklärung von Tarifverträgen (,Rüffert').

Einen Schritt grundsätzlicher ist die Forderung, die Entsenderichtlinie zu korrigieren. Die Diskussion darüber, ob die Entsenderichtlinie einen Maximalschutz (diese Deutung wird dem EuGH unterstellt) oder einen Mindestschutz der Arbeitnehmer vorsieht (so Generalanwalt Yves Bot in der Rs. ,Rüffert' und etliche Kritiker des Urteils), über den die Mitgliedstaaten hinausgehen könnten, verkennt indessen den Kern der Richtlinie: Ihr geht es um Gleichstellung der entsandten Arbeitnehmer mit den Einheimischen in bestimmten Bereichen - die Entgegensetzung von Dienstleistungsfreiheit und Arbeitnehmerschutz ist daher unzutreffend, es geht um beides. ${ }^{82}$ Daher ist eine Auslegung des Art. 3 Abs. 7 der Richtlinie - danach stehen die Abs. 1 bis 6 ,der Anwendung von für die Arbeitnehmer günstigeren Beschäftigungs- und Arbeitsbedingungen nicht entgegen“-, der zufolge die im Detail in Abs. 1 bis 6 niedergelegten Mindestbedingungen durch diese völlig offene Regelung wieder negiert werden können, kaum plausibel. ${ }^{83} \mathrm{Zu}$ Recht wird hier auf Erwägungsgrund 17 der Richtlinie hingewiesen, ${ }^{84}$ wonach die im Gastland geltenden zwingenden Bestimmungen über ein Mindestmaß an Schutz nicht der „Anwendung von Arbeitsbedingungen“, die für die Arbeitnehmer günstiger sind, entgegenstehen dürfen.

Eine Konkretisierung der Richtlinie wäre aber in der folgenden Richtung denkbar: Man müsste in die Richtlinie hineinschreiben, dass auch bei nicht für verbindlich erklärten Tarifverträgen eine Bindung bestehen soll. Die Vereinbarkeit mit dem Primärecht wäre dann zwar immer noch zu prüfen, aber im Ergebnis wohl zu bejahen. Die Beschränkung der Tariftreueklausel auf die öffentlichen Aufträge findet der EuGH nicht geeignet für das Ziel des Arbeitnehmerschutzes, insoweit fordert er einmal mehr „gesetzgeberische Konsequenz“ ein: ${ }^{85}$ Wenn der Arbeitnehmerschutz so wichtig ist, dann muss er für alle gelten: „Auch in Europa ist es Aufgabe des Gesetzgebers, für den Ausgleich kollidierender Rechtsgüter zu sorgen". 86

Der Hinweis auf eine Änderung der Entsenderichtlinie macht indessen sogleich deutlich, worin das Problem bei den soeben skizzierten einfachen Lösungen besteht: Sie erfordern po-

80 BVerfG, Urteil vom 11.07.2006, 1 BvL 4/00, BVerfGE 116, 202. Siehe auch BayVerfGH, Entscheidung vom 20.06.2008, in: Europäische Zeitschrift für Wirtschaftsrecht 2008, S. 675-679.

81 Diese Kritik kann hier nicht in allen Einzelheiten nachgezeichnet werden.

82 Klumpp: Dienstleistungsfreiheit vs. Tariftreue, 2008, S. 3475-3476. Siehe auch Reich: Free Movement v. Social Rights, 2008, S. 151, der unterstreicht, dass die Entsenderichtlinie aus rechtssystematischen Gründen nicht auf die üblichen Löhne in einem Aufnahmestaat abzielen kann, sondern wirklich Mindestlöhne meinen muss, will man nicht die Regelungsgrundlage der Richtlinie infrage stellen.

83 Becker: Anmerkung zur Entscheidung Rüffert, 2008, S. 892.

84 Ebenda.

85 Ebenda, S. 893.

86 Ebenda. 
litische Mehrheiten, teils in den Mitgliedstaaten, teils auf europäischer Ebene, die derzeit nicht in Sicht sind - ob die 1996 - übrigens gegen Portugal und Großbritannien - beschlossene Richtlinie in der heutigen, um 12 Mitgliedstaaten größeren Europäischen Union überhaupt eine Mehrheit fände, ist bereits fraglich. Insbesondere lässt sich fragen, warum die Mitgliedstaaten mit Lohnkostenvorteilen diesen Vorteil preisgeben sollten, solange er noch besteht. ${ }^{87}$

Eine Reihe von Einzelpunkten aus den Urteilen verdient eine intensivere Betrachtung:

Horizontale Wirkung der Grundfreiheiten? Grundfreiheiten gelten normalerweise nur gegenüber der öffentlichen Gewalt und nicht zwischen Privaten. In der Rs. ,Viking ' erläutert der EuGH allerdings, dass - weil die Arbeitsbedingungen in den verschiedenen Mitgliedstaaten teilweise durch Gesetze oder Verordnungen und teilweise durch Tarifverträge und sonstige Maßnahmen, die von Privatpersonen geschlossen beziehungsweise vorgenommen werden, geregelt sind - die Gefahr bestehe, dass eine Beschränkung der in den genannten Artikeln vorgesehenen Verbote auf Maßnahmen der öffentlichen Gewalt bei ihrer Anwendung zu Ungleichheiten führe.

Der Sache nach schließt der EuGH hier an die Rechtsprechung zur Bindung von Sportverbänden seit 1974 an. ${ }^{88}$ Und wahrscheinlich ist die Argumentation, die auf die Macht, kollektive Regelungen zu setzen, abstellt und hinter der das Argument gesellschaftlicher Macht im Allgemeinen steht, durchaus von den mächtigen Sportverbänden auf mächtige Gewerkschaftsverbände übertragbar. Gleichwohl ist die Einbeziehung von nichtstaatlichen Akteuren in die Bindungen durch die Grundfreiheiten eine der angreifbarsten Stellen der EuGHRechtsprechung, weil das gesamte System der Rechtfertigungsmöglichkeiten für Beeinträchtigungen von Grundfreiheiten auf Staaten - konkret: auf Wahrung von Gemeinwohlbelangen - zugeschnitten ist und nicht auf Private. ${ }^{89}$

Der EuGH hat freilich in einzelnen Bereichen ohnehin die Bindung nicht nur des Staates, sondern auch Privater an das Europarecht weiter vorangetrieben. ${ }^{90}$ Die perspektivisch danach denkbare Bindung grenzüberschreitend tätiger Konzerne (wie etwa der „Deutschen Telekom") an die Grundfreiheiten des EGV erscheint danach jedenfalls nicht ausgeschlossen und wäre das Korrelat zur Bindung der Gewerkschaften.

Unzureichende Sozialsensibilität des EuGH? Eine unzureichende Sozialsensibilität lässt sich dem EuGH letztlich nicht vorwerfen. ${ }^{91}$ So heißt es in der Rs. ,Viking' in Rn. 77, dass das Recht auf Durchführung einer kollektiven Maßnahme, die den Schutz der Arbeitnehmer zum Ziel hat, ein berechtigtes Interesse darstellt, das grundsätzlich eine Beschränkung einer der vom Vertrag gewährleisteten Grundfreiheiten rechtfertigen kann und dass der Schutz der Arbeitnehmer zu den bereits vom EuGH anerkannten zwingenden Gründen des Allgemeininteresses zählt.

Schließlich stellt der Gerichtshof ausdrücklich klar: „,[D]ie Tätigkeit der Gemeinschaft [umfasst, d. Verf.] nach dem Wortlaut von Art. 3 Abs. 1 Buchst. c und j EG nicht nur, einen Binnenmarkt, der durch die Beseitigung der Hindernisse für den freien Waren-, Personen-, Dienstleistungs- und Kapitalverkehr zwischen den Mitgliedstaaten gekennzeichnet ist ${ }^{*}$, sondern auch, eine Sozialpolitik‘ [...]. Art. 2 EG bestimmt nämlich, dass es u. a. Aufgabe der

87 Ebenda.

88 EuGH Rs. 36/74 (Walrave und Koch), Slg. 1974, 1405; EuGH Rs. C-415/93 (Bosman), Slg. 1995 , I-4921.

89 Vgl. dazu auch Loïc Azoulai: The Court of Justice and the Social Market Economy: The Emergence of an Ideal and the Conditions for its Realization, in: Common Market Law Review 2008, S. 1335-1355.

90 EuGH Rs. C-281/98 (Angonese), Slg. 2000, I-4139.

91 So auch im Ergebnis das Bundesverfassungsgericht im Lissabon-Urteil, BVerfG, Urteil vom 30.06.2009 - Lissabon, Absatz-Nr. 392ff. 
Gemeinschaft ist, , eine harmonische, ausgewogene und nachhaltige Entwicklung des Wirtschaftslebens" sowie ,ein hohes Beschäftigungsniveau und ein hohes Maß an sozialem Schutz“ zu fördern.“ „Da die Gemeinschaft somit nicht nur eine wirtschaftliche, sondern auch eine soziale Zielsetzung hat, müssen die sich aus den Bestimmungen des Vertrags über den freien Waren-, Personen-, Dienstleistungs- und Kapitalverkehr ergebenden Rechte gegen die mit der Sozialpolitik verfolgten Ziele abgewogen werden, zu denen, wie aus Art. 136 Abs. 1 EG hervorgeht, insbesondere die Verbesserung der Lebens- und Arbeitsbedingungen, um dadurch auf dem Wege des Fortschritts ihre Angleichung zu ermöglichen, ein angemessener sozialer Schutz und der soziale Dialog zählen. “92

Auch in der ,Rüffert'-Entscheidung nennt der EuGH mögliche Rechtfertigungsgründe für eine Einschränkung der Marktfreiheiten, hier der Dienstleistungsfreiheit: ,,Schutz der Arbeitnehmer, Schutz der autonomen Ordnung des Arbeitslebens durch Koalitionen“ und „die finanzielle Stabilität der sozialen Versicherungssysteme“. Um den Schutz des Tarifvertrags als Regelungsinstrument ${ }^{93}$ geht es in der ,Rüffert'-Entscheidung aus Sicht der Betroffenen aber übrigens nicht, sondern um den Schutz eines bestimmten Tarifergebnisses. ${ }^{94}$

Negiert der EuGH das Streikrecht? Das Gegenteil ist der Fall. ${ }^{95}$ Der EuGH hat in der Rs. ,Laval' das Streikrecht als europäisches Grundrecht anerkannt, das freilich im Einzelfall mit den Grundfreiheiten des EGV abzuwägen ist. Auch das deutsche Grundgesetz gewährleistet das Streikrecht nicht ohne jede Schranke.

In der Rs. ,Laval“ heißt es, ,das Recht auf Durchführung einer kollektiven Maßnahme einschließlich des Streikrechts [ist, d. Verf.] als Grundrecht anzuerkennen, das fester Bestandteil der allgemeinen Grundsätze des Gemeinschaftsrechts ist, deren Beachtung der Gerichtshof sicherstellt [...]." ${ }^{\text {96 }}$ Die dann folgende Passage ändert daran nichts. Zwar heißt es dort: ,[Es, d. Verf.] genügt der Hinweis, dass es den Mitgliedstaaten in den Bereichen, für die die Gemeinschaft nicht zuständig ist, zwar grundsätzlich weiterhin freisteht, die Bedingungen für den Bestand der fraglichen Rechte und die Modalitäten ihrer Ausübung festzusetzen, dass sie aber gleichwohl gehalten sind, das Gemeinschaftsrecht bei der Ausübung dieser Befugnis zu beachten. “97 Damit zieht der EuGH aber nur die allgemein anerkannten Kriterien für die Beurteilung der Rechtmäßigkeit von Einschränkungen durch nationales Recht heran.

Ferner wiederholt er die schon aus den Urteilen ,Schmidberger ${ }^{998}$ und ,Omega 999 bekannte Formel, der zufolge ,der Grundrechtsschutz ein berechtigtes Interesse ist, das grundsätzlich geeignet ist, eine Beschränkung der Verpflichtungen zu rechtfertigen, die nach dem Gemeinschaftsrecht, auch kraft einer durch den EG-Vertrag gewährleisteten Grundfreiheit [...] bestehen. " 100

92 EuGH Rs. C-438/05 (Viking), Slg. 2007, I-10779, Rn. 78-79; EuGH Rs. C-341/05 (Laval), Slg. 2007, 11767, Rn. 104-105.

93 In diese Richtung Eva Kocher: Die Tariftreueerklärung vor dem EuGH, in: Der Betrieb 2008, S. 1042-1045, hier S. 1045.

94 Zutreffend Klumpp: Dienstleistungsfreiheit vs. Tariftreue, 2008, S. 3477.

95 Dies hält auch das Bundesverfassungsgericht im Lissabon-Urteil fest, BVerfG, Urteil vom 30.06.2009 - Lissabon, Absatz-Nr. 398.

96 EuGH Rs. C-438/05 (Viking), Slg. 2007, I-10779, Rn. 44

97 Ebenda, Rn. 40.

98 EuGH Rs. C-112/00 (Schmidberger), Slg. 2003, I-5659.

99 EuGH Rs. C-36/02 (Omega), Slg. 2004, I-9609.

100 EuGH Rs. C-438/05 (Viking), Slg. 2007, I-10779, Rn. 45. 
Gibt es ein Wahrnehmungsproblem? Wenn die Zusammenhänge so sind, wie bis hierher geschildert, dann erscheint die Intensität der Kritik besonders erstaunlich. Dies wirft die Frage auf, ob es möglicherweise schlicht ein Wahrnehmungsproblem angesichts der Rechtsprechung des EuGH gibt, weil die Details der Rechtsprechung nicht mehr einfach erklärbar und verständlich erscheinen. Sicherlich ist zuzugestehen, dass die Entscheidungen komplex sind, weil die infrage stehende Rechtslage komplex ist. Daraus aber beispielsweise abzuleiten, dass der EuGH sich in den hier in Rede stehenden Fragen eine Selbstbeschränkung auferlegen sollte, eine Art ,social self restraint', wäre wohl nicht angemessen. In Anbetracht der oben skizzierten Interessensgegensätze zwischen alten und neuen Mitgliedstaaten, die hinter den Fällen stehen, kann der EuGH sich allenfalls dann, wenn es keine Anhaltspunkte für die protektionistischen Motivationen gibt, zurücknehmen. Genau dies ist aber in der Rs. , Viking ' der Fall gewesen, bei deren Bearbeitung der EuGH das durch einen europaweit organisierten Gewerkschaftsverband vorgebrachte Argument durch das nationale Gericht prüfen und entscheiden ließ. Diese Besonderheit des, Viking '-Falls wird in der Diskussion nicht selten übersehen.

Dem Wahrnehmungsproblem lässt sich entgegenwirken, indem die Interessenlagen klar angesprochen und die Konsequenzen der verschiedenen Argumentationslinien deutlich gemacht werden: Es geht gar nicht so sehr um den EuGH. Es geht vielleicht in weiten Teilen um Frustrationsabbau im Angesicht der immer weniger beeinflussbaren ökonomischen Verhältnisse, für den der EuGH sich als Ziel besonders eignet, weil er außerhalb des eigenen politischen Bezugssystems steht (Sündenbockfunktion). Es geht möglicherweise auch schlicht um die im Rahmen des geltenden Europarechts ausgetragenen Interessensgegensätze bis hin zu ökonomischen Verteilungskämpfen zwischen den alten und den neuen Mitgliedstaaten. Es geht auch um die Frage, ob man die Grundfreiheiten weiter ernst nehmen will oder ob man in nationale Egoismen zurückfällt. Gerade letztere Frage wird sich in Zeiten zunehmender ökonomischer Probleme immer drängender stellen. ${ }^{101}$

\section{Perspektiven und Spielräume - der EuGH und das soziale Europa}

Aus den oben gemachten Beobachtungen lassen sich Schlussfolgerungen zu Perspektiven und Spielräumen des EuGH und für den EuGH im Themenfeld ,Soziales Europa' ableiten. Institutionelle Folgerungen im Sinne einer Veränderung der institutionellen Rolle des EuGH sind dabei wenig perspektivreich. Wer die Rechtsprechung beanstandet, der muss das Recht ändern: sei es auf der Mikroebene der Rechtsetzung wie beispielsweise der Entsenderichtlinie oder auf der Makroebene der Grundfreiheitenstruktur beziehungsweise Erweiterungsvoraussetzungen. Es zeigt sich, dass der EuGH nur begrenzt Akteur oder gar Motor der sozialen Integration sein kann.

\section{Institutionelle Folgerungen}

Der EuGH ist letztlich nur so gut oder schlecht wie das Europarecht, das er auszulegen und fortzuentwickeln hat. Die Kritik am EuGH als Institution geht daher schon im Ansatz in die falsche Richtung. Die Institution des EuGH steht nicht zur Disposition. Die bei Kritik am EuGH immer wieder hervorgeholten Konzepte eines neu zu schaffenden europäischen Kompetenzgerichts oder Ähnliches ${ }^{102}$ erweisen sich bei näherem Nachdenken als nutzlos.

101 Dann wird auch zwischen den alten Mitgliedstaaten der protektionistisch-egoistische Reflex wieder aufbrechen. Siehe zwischen Italien und Großbritannen BBC: Refinery strikes spread across UK, 30.01.2009, abrufbar unter: http://news.bbc.co.uk/1/hi/uk/7859968.stm (letzter Zugriff: 18.06.2009).

102 Siehe zuletzt etwa den Beitrag von Gerken (mit Herzog): Stoppt den Europäischen Gerichtshof!, 2008. In diesem Beitrag fällt übrigens auf, dass ausgerechnet die Urteile ,Viking', ,Laval' etc. nicht beanstandet werden, wohl weil diese als arbeitgeberfreundliche missverstanden wurden. Zugleich besteht damit ein weiterer Beleg dafür, dass Kritik von allen Seiten darauf hindeutet, dass der EuGH sich auf einem Mittelweg befindet. 
Die Forderung nach einer politischen Kontrolle des EuGH ${ }^{103}$ geht nicht nur von der unzutreffenden Prämisse eines ,liberalen Programms" 104 beim EuGH aus, sie würde die in der EuGH-Rechtsprechung tatsächlich bestehende Balance zwischen Markt und Sozialem zudem gerade den Zufälligkeiten politischer Mehrheitsbildung im Europäischen Rat überlassen, sodass eine echte radikale Deregulierung und Liberalisierung nicht einmal mehr durch das Recht gezähmt wäre.

Frontale Richterschelte ist gegenüber dem EuGH noch weniger hilfreich. Der gelegentlich unterbreitete Vorschlag, den EuGH als politischen Akteur anzusehen, mit einer bestimmten eigenen Agenda, und ihn entsprechend politisch anzugehen, geht an der Realität der alles andere als monolithischen Institution ohnehin völlig vorbei. Ab einem bestimmten Punkt und in einer bestimmten Form ist politische Richterschelte - nicht die sachlich-fachliche Kritik, die selbstverständlich möglich sein muss - ein Ausweis unzureichender Rechtsstaatssensibilität: Weil sich Richter gegen politische Vorwürfe nicht öffentlich wehren können, sind politische Angriffe auf Gerichte und Urteile ab einem bestimmten Grad ein äußerst gefährliches Spiel.

Frontale politische Angriffe auf den EuGH zum Beispiel im Wahlkampf ${ }^{105}$ legen die Axt an das Verständnis der europäischen Integration als Rechtsgemeinschaft. Es lässt sich beobachten, dass fast nur in Deutschland - kaum unmittelbar von den fraglichen Fällen in Schweden, Finnland, Estland und Lettland betroffen - von Gewerkschaftsseite fast schon kampagnenartig auf den EuGH losgegangen wird. Dabei hat der Grundkonsens über den Vorrang des Rechts in der europäischen Integration gerade Deutschland, das als größter Mitgliedstaat grundsätzlich unter Hegemonieverdacht steht, gute Dienste geleistet. Hier stehen sowohl Parteien wie auch Gewerkschaften in der Verantwortung, in der Hoffnung auf kurzfristige Wählersympathien populistische Vereinfachungen der europäischen Zusammenhänge nicht zu übertreiben. Die Integrität der Rechtsgemeinschaft und der europäischen Gerichtsbarkeit lässt sich, einmal zerstört, nicht ohne Weiteres wieder herstellen.

Kritik am EuGH durch bestimmte Interpretations- und durch Interessengemeinschaften folgt einem wiederkehrenden Muster: die Verfassungsrechtler, die Verwaltungsrechtler, die Zivilrechtler und jüngst die Strafrechtler mussten die Erfahrung machen, dass das Europarecht ihren Bereich nicht unberührt lässt und haben sich nach anfänglichen Protesten, bis hin zum Widerstand, nach einiger Zeit mit diesem Umstand arrangiert. Jenseits der juristischen Interpretationsgemeinschaften sind die Sportverbände, die zunächst vehement die Einwirkung des Europarechts auf ihren Bereich bekämpften, ein Beispiel für diesen Verlauf. Die Gewerkschaften weisen freilich in etlichen Mitgliedstaaten die Besonderheit auf, dass sie in viel größerem Maße als etwa die Sportverbände eine Politisierung ihrer Sicht durchsetzen und beispielsweise auch politische Parteien stärker zu instrumentalisieren versuchen können. Gleichwohl dürfte auch hier die Perspektive einer zunehmenden Akzeptanz der europäischen Dimension mit ihrer spezifischen Einwirkungslogik nicht unrealistisch sein.

Banal ist der Hinweis darauf, dass die Rechtsprechung des EuGH davon abhängt, welche Personen im Gericht tätig sind. Dies berührt das Thema der Richterauswahl und einer möglichen Vorabklärung der Haltungen von Richterkandidaten beispielsweise zum Thema, Soziales Europa'. Ein weiterer Faktor ist schließlich mittlerweile die Heterogenität des Gerichts, in der sich die Anzahl der Mitgliedstaaten spiegelt. Will man an der Institution Veränderungen vornehmen, um die Brisanz bestimmter Urteile besser hervortreten zu lassen, dann ließe

103 Fritz W. Scharpf: Legitimacy in the Multilevel European Polity, MPIfG Working Paper 09/1.

104 Ebenda.

105 In den Wahlkämpfen des Jahres 2009 sind entsprechende populistische Vereinfachungen zu beobachten. 
sich allenfalls über die Einführung von Sondervoten nachdenken, die bisher nicht bestehen. Hier würden sich möglicherweise dann Konflikte auch innerhalb des Gerichts deutlicher nachvollziehen lassen, um den Preis freilich des nach außen einheitlichen Auftretens des Gerichts. Möglicherweise wird die Funktion von Sondervoten freilich schon durch die Generalanwälte übernommen, wenn diese in ihren Schlussanträgen vom späteren Urteil abweichende Einschätzungen und Empfehlungen geben. ${ }^{106}$

\section{Die Mikroebene der Rechtsetzung}

Wer mit der Rechtsprechung nicht einverstanden ist, muss also versuchen, das Europarecht zu ändern, anstatt die Institution anzugreifen. Das betrifft auf einer Mikroebene die Entsenderichtlinie, die in drei der vier in der jüngsten Zeit in der Diskussion stehenden EuGH-Entscheidungen eine Rolle spielt. Dabei ist freilich zu beachten, dass die Entsenderichtlinie ihrerseits mit dem Primärrecht vereinbar sein muss. Gerade deswegen ist von Bedeutung, dass der EuGH im Grundsatz den Arbeitnehmerschutz als Rechtfertigung zur Beschränkung der Grundfreiheiten anerkannt hat, sodass mit der Entsenderichtlinie entsprechende Ausnahmen zur Dienstleistungsfreiheit überhaupt möglich sind.

\section{Die Makroebene der Grundfreiheitenstruktur}

Es steht den Mitgliedstaaten stets die Möglichkeit offen, EuGH-Urteile durch Änderung der Verträge zu korrigieren. Dies ist in der Vergangenheit durchaus erfolgt. ${ }^{107}$

Auf einer allgemeineren Betrachtungsebene lässt sich die Frage stellen, ob die durch den EuGH entwickelte Rechtsstruktur im Bereich der Grundfreiheiten sich nicht zu sehr von dem, worum es eigentlich bei Grundfreiheiten und Binnenmarkt gehen sollte, entfernt hat. ${ }^{108}$ Konkret ist dies die Frage, ob mit Blick auf die Abwehr von Protektionismus ein Verständnis der Grundfreiheiten als Beschränkungsverbote - und nicht als bloße Diskriminierungsverbote - womöglich zu weit geht. Diskriminierungsverbote fordern im Grundsatz lediglich die Gleichbehandlung mit Inländern, Beschränkungsverbote dagegen entsprechen echten Freiheitsrechten und fragen nach der Beeinträchtigung bei der Ausübung einer Grundfreiheit, unabhängig davon, wie Inländer behandelt werden. Das Problempotenzial eines Beschränkungsverbotes ist dabei, dass tendenziell jeder Unterschied zwischen den Rechtsordnungen zunächst als Problem interpretiert werden kann. Der EuGH hat im Bereich des Warenverkehrs dies so gelöst, dass er nach der ,Keck'-Rechtsprechung bestimmte mitgliedstaatliche Maßnahmen von vornherein aus dem Anwendungsbereich des Europarechts herausnimmt. ${ }^{109}$ Im Kern geht es dabei um sozio-ökonomische Besonderheiten. Allerdings ist es dafür eine Voraussetzung, dass die mitgliedstaatlichen Maßnahmen diskriminierungsfrei operieren. Dies war in der Rs. ,Laval' gerade nicht der Fall. Aber auch unabhängig davon ist nicht in Sicht, dass der EuGH seine Konzeption der Grundfreiheiten auf bloße Diskriminierungsverbote zurückfährt.

Beachtung verdient in diesem Kontext indessen nochmals die Besonderheit in der Rs. ,Viking': Dort wurden die Beschränkungen durch den europäischen Gewerkschaftsdachverband auf europäischer Ebene bewirkt, was die Vermutung zumindest nahelegt, dass es eher

106 So beispielsweise auch Generalanwalt Miguel Maduro in der Rechtsache, Viking`. Siehe EuGH Rs. C-438/05 (Viking), Slg. 2007, I-10779.

107 Siehe das sogenannte ,Barber-Protokoll‘, Protokoll (Nr. 17) zu Art. 141 des Vertrags zur Gründung der Europäischen Gemeinschaft, 2006, zu EuGH Rs. 262/88 (Barber), Slg. 1990, I-1889.

108 Kritisch z. B. Thorsten Kingreen: Grundfreiheiten, in: Armin von Bogdandy (Hrsg.): Europäisches Verfassungsrecht, 2. Aufl., i. E.

109 EuGH verb. Rs. C-267/91 und C-268/91 (Keck und Mithouard), Slg. 1993, 6097. 
nicht um Protektionismus oder nationalen Wirtschaftsegoismus ging. Folgerichtig überließ der EuGH die Entscheidung dem vorlegenden Gericht.

Eine andere grundsätzlich-strukturelle Überlegung betrifft die Frage, ob die in der Charta der Grundrechte verankerten und damit gegenüber dem Richterrecht sichtbareren sozialen Grundrechte den Mitgliedstaaten bei der Geltendmachung von Ausnahmen zu den Grundfreiheiten im Anwendungsbereich des Europarechts weitere Schranken setzen werden. Bei hier denkbaren Konflikten zwischen Grundfreiheiten der einen und (sozialen) Grundrechten der anderen wird der EuGH Gelegenheit haben, die soziale Komponente des europäischen Rechts weiter zu entwickeln.

\section{Die Makroebene der Erweiterungsstrategie}

Zu guter Letzt veranlasst der Hintergrund der Rs. ,Viking', ,Laval' und ,Rüffert' zu der Überlegung, dass für künftige Beitritte die derzeitigen Probleme mit Mitgliedstaaten sehr unterschiedlicher ökonomischer Entwicklungsstände Lehren sein sollten. ${ }^{110}$ Das Binnenmarktkonzept erträgt offenbar ökonomische Divergenz zwischen den Mitgliedstaaten nur begrenzt. $\mathrm{Zu}$ überlegen wäre in diesem Kontext, ob nicht das Solidaritätsprinzip in die Selbstbeschreibung der Europäischen Union in Art. 6 Abs. 1 EUV aufgenommen werden müsste. Damit wäre nicht nur für den Fall einer gravierenden Verletzung des Sozialprinzips die Drohkulisse der Sanktionen nach Art. 7 EUV denkbar. Es wäre auch sichergestellt, dass Sozialstaatlichkeit Beitrittskriterium ist. ${ }^{111}$ In Art. 2 EUV-Liss., der Nachfolgebestimmung zu Art. 6 EUV, findet Solidarität immerhin Erwähnung.

\section{Schlussbetrachtung}

Insgesamt ergibt sich, dass der EuGH nur begrenzt prospektiv Akteur der sozialen Integration in der Europäischen Union sein kann. Er hat das bestehende Europarecht auszulegen und weiterzuentwickeln. Damit kommt es für ein Mehr an sozialem Europa auf die Weiterentwicklung des Rechts an, und dies erfordert eben entsprechende Mehrheiten und bestimmte sozio-ökonomische Konstellationen in und zwischen den Mitgliedstaaten. Recht und Institutionen setzen einen Rahmen und schaffen Voraussetzungen für Politik, sie ersetzen diese nicht.

Angriffe auf den EuGH, wie sie im Kontext der hier diskutierten Entscheidungen zu beobachten sind, bergen Risiken für das Konzept der Rechtsgemeinschaft, soweit sie den EuGH beschädigen können und ihm eine Rolle zuweisen, die er nicht ausfüllen kann: Der EuGH hat nicht die Primärverantwortung für das soziale Europa.

An vielen Stellen hat der EuGH immerhin Spielräume genutzt oder geschaffen, die als soziale Abfederung des Binnenmarktes wirken und hat damit soziales Europa für den Einzelnen greifbar gemacht, wie oben gezeigt worden ist. ${ }^{12}$ Viel mehr kann man von einem Gericht vielleicht erträumen, aber nicht verlangen.

110 Dazu, dass vergleichbare Probleme auch bei vergangenen Beitrittsrunden aufkamen, siehe etwa EuGH Rs. C-113/89 (Rush Portuguesa Lda/Office National d'Imigration), Slg. 1990, I-1417. Die Entscheidung war übrigens Ausgangspunkt für die Ausarbeitung einer Entsenderichtlinie.

111 Der Vertrag von Lissabon nennt immerhin in Art. 2 EUV Solidarität im Kontext der Werte der Union.

112 Siehe auch als jüngeres Beispiel das Urteil des EuGH vom 20. Januar 2009 zum Urlaubsanspruch bei Krankheit (bejaht). EuGH verb. Rs. C-350/06 und C-520/06 (Schultz-Hoff/Deutsche Rentenversicherung und Stringer u. a./Her Majesty's Revenue and Customs). 